\title{
Investigations on Tl-2223 thin films fabricated through ultrasonic spray pyrolysis under oxygen deficient conditions
}

\author{
H K SINGH ${ }^{\dagger}$ and O N SRIVASTAVA* \\ Department of Physics, Banaras Hindu University, Varanasi 221 005, India \\ †Present Address: Department of Physics, SGR Post Graduate College, Dobhi, Jaunpur 222 149, India
}

MS received 14 September 1999; revised 27 July 2000

\begin{abstract}
In this paper we have shown that polycrystalline films corresponding to Tl-2223 phase can be grown by employing high thalliation temperatures and short thalliation times. Ultrasonically deposited precursor films corresponding to $\mathrm{Ba}_{2} \mathrm{Ca}_{2.2} \mathrm{Cu}_{3.3} \mathrm{O}_{x}\left(\mathrm{Ag}_{y}\right)$ have been thalliated under high vacuum $\left(\sim 10^{-5}\right.$ torr $)$ at $890^{\circ} \mathrm{C}$ to obtain single phase $\mathrm{Tl}-2223$ films. An off-stoichiometric and unreacted pellet of composition $\mathrm{Tl}_{2.05} \mathrm{Ba}_{2} \mathrm{Ca}_{2} \mathrm{Cu}_{3} \mathrm{O}_{z}$ has been used as source of $\mathrm{Tl}$. We have shown that oxygen ambient is not necessary for the growth of Tl-2223 phase. The as-thalliated films have $T_{\mathrm{c}}$ 's in the range $123 \mathrm{~K} \pm 0.70 \mathrm{~K}$. The $T_{\mathrm{c}}$ has been found to be independent of the addition of $\mathrm{AgNO}_{3}$ to the precursor. The zero field transport $J_{\mathrm{c}}$ has been observed to be $>1.2 \times 10^{5} \mathrm{~A} / \mathrm{cm}^{2}$ at $77 \mathrm{~K}$. Near $T_{\mathrm{c}}(110 \mathrm{~K}-122 \mathrm{~K}), J_{\mathrm{c}}$ has been observed to follow the power law $J_{\mathrm{c}} \propto$ $\left(1-T / T_{\mathrm{c}}\right)^{p}, p \approx 2$. A power law with $p \approx 1.4$ has been observed for the temperature range $70 \mathrm{~K}-110 \mathrm{~K}$. An optimum doping of $\mathrm{Ag}$ has been observed to induce about $25 \%$ increase in $J_{\mathrm{c}}$ and it also leads to uniform and enlarged grain growth. The surface morphology of Ag free samples contains plate like grains having arbitrary shapes. In contrast to this $0 \cdot 35 \mathrm{Ag}$ doped sample exhibits nearly rectangular plate like grains.
\end{abstract}

Keywords. HTSC; thin films; Tl-2223; ultrasonic spray pyrolysis.

\section{Introduction}

One of the high temperature superconducting cuprates (HTSCs) which has an inherent potential for practical applications, especially at temperatures close to $120 \mathrm{~K}$ is the $\mathrm{Tl}$ bilayer phase $\mathrm{Tl}_{2} \mathrm{Ba}_{2} \mathrm{Ca}_{2} \mathrm{Cu}_{3} \mathrm{O}_{10}$ (Tl-2223). In bulk form this superconducting cuprate has a transition temperature $\left(T_{\mathrm{c}}\right)$ as high as $128 \mathrm{~K}$ (Liu et al 1992) and in thin film form, it has been found to be superconducting up to $122 \mathrm{~K}$ (Nabatame et al 1990). In addition to high $T_{\mathrm{c}}$, Tl2223 films have been observed to exhibit fairly high transport critical current density $\left(J_{\mathrm{c}}\right)$ and in fact $J_{\mathrm{c}} \sim 10^{6}$ $\mathrm{A} / \mathrm{cm}^{2}(77 \mathrm{~K}, 0 T)$ has been reported (Lee et al 1990). One more advantage of Tl-2223 films is that they are more stable and easier to synthesize than the recently discovered Hg bearing HTSC films having $T_{\mathrm{c}} \sim 125 \mathrm{~K} \pm 5 \mathrm{~K}$. Thus, for various microelectronic/electronic applications especially near $120 \mathrm{~K}, \mathrm{Tl}-2223$ thin films seem to possess better potential.

The synthesis of good quality Tl-2223 thin films is very difficult especially single phase films due to highly volatile and toxic nature of $\mathrm{Tl}$ and its oxides. The in situ growth of Tl bearing HTSC thin films becomes more problematic. Till date only single $\mathrm{Tl}-\mathrm{O}$ layer phases $\mathrm{Tl}-$ 1212 and Tl-1223 have been grown in thin film form

*Author for correspondence employing in situ process (Mayers et al 1994). Consequently, an ex situ growth process consisting usually of the following three steps is employed: (i) deposition of $\mathrm{BaCaCuO}$ precursor film on desired substrate, (ii) incorporation of ' $\mathrm{Tl}$ ' into the precursor matrix (thalliation) and (iii) post thalliation annealing.

The deposition of precursor films has been carried out by various techniques, e.g. pulsed laser deposition (PLD), d.c. and rf sputtering, spray pyrolysis etc (Nabatame et al 1990; Lee et al 1990; Mayers et al 1994; Singh et al 1995; Lee et al 1989; Holstein and Parisi 1996). The incorporation of $\mathrm{Tl}$ into the precursor matrix, i.e. thalliation, has been achieved in two different ways, viz. (a) in closed assemblies having a desired amount of $\mathrm{O}_{2}$ pressure or a mixture of $\mathrm{O}_{2}$ and $\mathrm{N}_{2}$ or Ar gases (Lee et al 1990; Mayers et al 1994; Lee et al 1989; Holstein and Parisi 1996), and (b) in open reaction chambers fitted with oxygen inlet and outlet ports (Singh et al 1995). Higher thalliation temperatures $\left(\sim 890^{\circ} \mathrm{C}\right)$ are required to get the 2223 phase in closed reaction chambers (Lee et al 1989; Holstein and Parisi 1996), while the thalliation temperature is reduced in case of open reaction chambers provided with oxygen flow (Narain and Rukenstein 1989; Singh et al 1995). The thalliation time is also an important parameter and it has been observed that better quality films are grown employing longer thalliation time at lower temperature (Nabatame et al 1990). The third step of post thalliation annealing is carried out either in closed assem- 
blies (Nabatame et al 1990; Holstein and Parisi 1996) or in flowing oxygen (Singh et al 1995). It has been shown that $T_{\mathrm{c}}$ of bulk 2223 phase can be raised up to $128 \mathrm{~K}$ by very fine-tuning of the oxygen stoichiometry (Liu et al 1992). The oxygen partial pressure employed during the synthesis has been observed to affect the quality of Tl2223 bulk as well as films (Lee et al 1989). But no study seems to be available regarding the synthesis of Tl-2223 thin films under oxygen deficient conditions.

Of the various techniques used for the deposition of precursor films, spray pyrolysis, despite being a conventional and less sophisticated one, has been used quite successfully to fabricate Tl-2223 and Tl-1223 (de Luca et al 1993; Su et al 1994; Singh et al 1995). The synthesis of cuprate HTSC films using the spray pyrolysis technique has been reviewed by Jergel (1995). Recently synthesis methods and physical properties of Tl-bearing cuprates have been reviewed (Siegal et al 1997). More recently, this technique has been employed to synthesize $\mathrm{Hg}$ bearing HTSC films as well (Moriwaki et al 1996; Singh et al 1996). Spray pyrolysis deposited films of Tl-2223 and $\mathrm{Hg}(\mathrm{Tl})-1223$ have been used to fabricate rf SQUIDs (Khare et al 1994, 1997).

In this communication, we present the investigations on the oxygen deficient synthesis, zero field d.c. transport properties and microstructural features of $\mathrm{Tl}-2223$ thin films. Since a mild Ag doping $(\sim 0 \cdot 35)$ has been found to be conducive to higher critical currents (Singh et al 1995), we have, in addition to virgin Tl-2223 phase also investigated $0.35 \mathrm{Ag}$ doped Tl-2223 films. It has also been shown that better quality films can be grown employing higher thalliation temperatures and short thalliation times.

\section{Experimental}

In the present investigation synthesis of Tl-2223 thin films was carried out employing the following protocol. The $\mathrm{Ba}-\mathrm{Ca}-\mathrm{Cu}-\mathrm{O}$ precursor films corresponding to the composition $\mathrm{Ba}_{2} \mathrm{Ca}_{2 \cdot 3} \mathrm{Cu}_{3 \cdot 3} \mathrm{O}_{x}\left(\mathrm{Ag}_{y}\right) \quad(y=0 \cdot 00,0 \cdot 35)$ were deposited by spray pyrolysis employing an ultrasonic nebulizer. This technique involved deposition of $\mathrm{Tl}$ free precursor films from an aqueous solution on a desired substrate. Firstly an aqueous solution of the nitrates of the cation constituents was prepared. This solution was then converted into mist by an ultrasonic nebulizer operating at a frequency of $\sim 10^{6} \mathrm{~Hz}$. This mist was then made to impinge on a heated substrate resulting in formation of a film. This as-deposited film was then subjected to post deposition processing. The aqueous solution was prepared by dissolving metal nitrates $\mathrm{Ba}\left(\mathrm{NO}_{3}\right)_{2}, \mathrm{Ca}\left(\mathrm{NO}_{3}\right)_{2} 4 \mathrm{H}_{2} \mathrm{O}$, $\mathrm{Cu}\left(\mathrm{NO}_{3}\right)_{2} 3 \mathrm{H}_{2} \mathrm{O}$ and $\mathrm{AgNO}_{3}$ in requisite amounts in triple distilled water. The molarity of the aqueous solution was kept at $0.3 \mathrm{M}$ and the cationic ratio was $\mathrm{Ba} / \mathrm{Ca} /$ $\mathrm{Cu} / \mathrm{Ag}=2 / 2 \cdot 3 / 3 \cdot 3 / y$. Methanol was also added to the spray solution for reasons previously explained (Singh et al 1995). After homogenization with the aid of a hot plate and magnetic stirrer, the solution was sprayed on single crystal $\mathrm{MgO}(100)$ substrates $\left(1 \times 0.5 \times 0.1 \mathrm{~cm}^{3}\right)$. The ultrasonic nebulizer used for spraying was capable of generating a mist having average particle size of $\sim 1 \mu \mathrm{m}$. The substrate temperature was maintained at $\sim 150^{\circ} \mathrm{C}$. This deposition temperature is much less than the previously used temperature $\left(400-500^{\circ} \mathrm{C}\right)$ (Singh et al 1995). After deposition, the precursor films were slowly heated in air to $800^{\circ} \mathrm{C}$, kept there for $1 \mathrm{~h}$ and then slowly cooled to room temperature. The air annealed precursor films were then annealed in the presence of a $\mathrm{Tl}$ source to get the HTSC phase as follows. The Ag free film was placed in a $\mathrm{Pt}$ box, above this film a rectangular and unreacted pellet of composition $\mathrm{Tl}_{2.05} \mathrm{Ba}_{2} \mathrm{Ca}_{2} \mathrm{Cu}_{3} \mathrm{O}_{z}$ placed and finally the $0.35 \mathrm{Ag}$ admixed precursor film was placed. The box was then closed, kept in a quartz tube and evacuated to $>10^{-5}$ torr and then sealed. This tube was then kept in a tube furnace preheated to $890^{\circ} \mathrm{C}$ and the furnace temperature was maintained at $890^{\circ} \mathrm{C}$ for $2 \mathrm{~h}$ and was furnace cooled to room temperature. In the present case, we did not use external oxygen supply at any stage. The precursor film synthesis was carried out without any external oxygen supply and thalliation was carried out in high vacuum. This annealing schedule was different from previously used ones (Lee et al 1990; Nabatame et al 1990; Su et al 1994; Singh et al 1995; Holstein and Parisi 1996). Since, it is well established that 2223 phase in the TBCCO system is formed at $\sim 890^{\circ} \mathrm{C}$, we have used this as the thalliation temperature. Different thalliation time periods, viz. $1 \mathrm{~h}, 2 \mathrm{~h}, 3 \mathrm{~h}$ and $4 \mathrm{~h}$ were used in order to optimize the phase formation.

The as-grown samples were subjected to characterization by employing X-ray diffraction (XRD Philips PW 1710), transmission microscopy (TEM, Philips CM-12 coupled with EDAX), four-probe d.c. electrical characterization (Keithley Hall/resistivity set up) and microstructural characterization employing scanning electron microscopy (SEM Philips XL 20).

\section{Results and discussion}

The as-grown films are $\sim 1 \mu \mathrm{m}$ thick and usually have cationic composition very close to the envisaged one. Both, Ag free and 0.35 Ag doped samples have glossy appearance. The lower temperature used for the deposition of precursor films $\left(\sim 150^{\circ} \mathrm{C}\right)$ has been observed to be conducive to denser film growth and increase in the deposition temperature leads to porosity as well as surface roughness of films.

The analysis of XRD data of the as-grown samples shows that $1 \mathrm{~h}$ thalliated film is biphasic with a mixture of 2223 and 2212 phases; 2223 being the majority phase. The 3 and $4 \mathrm{~h}$ thalliated films are also biphasic with 2223 
as the majority phase and 1223 as the minority phase. The $2 \mathrm{~h}$ thalliated film shows single phase 2223. This holds true for both $\mathrm{Ag}$ free as well as 0.35 Ag doped samples. This means that $0.35 \mathrm{Ag}$ doping has no effect on the phase formation in the present case. Since the films thalliated at $890^{\circ} \mathrm{C}$ for $2 \mathrm{~h}$ have been observed to be single phase 2223, further investigations were performed only on these films. Representative XRD patterns of Ag free and $\mathrm{Ag}$ doped films are shown in figure 1. The XRD patterns show that our samples are highly $c$-axis oriented as evidenced by $00 \mathrm{l}$ reflections. As marked in figure $1 \mathrm{~b}$ in case of Ag doped films some reflections corresponding to silver have also been observed. In addition to the $00 l$ reflections, some peaks indicative of reflection from lattice planes other than $00 l$ have also been observed. The additional peaks are also indexed in figure 1 . The lattice parameters of the tetragonal unit cell as calculated from the XRD data have been found to be $a=3.854 \AA$ and $c=35.725 \AA$. No observable change in these lattice parameters has been noticed as a consequence of Ag doping. The absence of any other impurity phase (barring Ag in Ag doped samples) shows that our samples are of high degree of purity. All these structural features have been corroborated by local area investigations carried out by TEM on these samples. In fact, electron diffraction and direct lattice imaging have yielded the unit cell parameters to be $a=3.85 \AA$ and $c / 2=17.9 \AA$. Representative selected area electron diffraction pattern and the corresponding lattice image are shown in figure 2 . The resistance versus temperature $(R-T)$ behaviour of the as-grown $\mathrm{Ag}$ free and $0.35 \mathrm{Ag}$ doped films is shown in figure 3, where the normalized resistance is plotted against temperature. The normal state in both the cases is highly metallic and free of any fluctuations. The onset of transition is around $126 \mathrm{~K}$ in both $\mathrm{Ag}$ free and $\mathrm{Ag}$ doped samples and zero resistance is observed at $123.7 \mathrm{~K}$ and 123.6 K in $\mathrm{Ag}$ free and 0.35 Ag admixed samples respectively. The $T_{\mathrm{c}}$ of all the films prepared in the present case to test the reproducibility of our process has been found to lie in the range $122.7 \mathrm{~K}<T_{\mathrm{c}}<123.7 \mathrm{~K}$. This shows that our synthesis protocol is efficiently optimized and reproducible. It will also be taken into consideration that the observed $T_{\mathrm{c}}$ values are higher than previously reported values (Holstein and Parisi 1996; Siegal et al 1997).

The present investigation leads to another interesting observation that oxygen ambient is not necessary for the growth of higher $T_{\mathrm{c}}$, single phase Tl-2223 when nitrates of $\mathrm{Ba}, \mathrm{Ca}, \mathrm{Cu}$ and $\mathrm{Ag}$ are used as starting materials. This is well evidenced by the fact that films having $T_{\mathrm{c}} \mathrm{s}$ close to $124 \mathrm{~K}$ have been grown under oxygen deprived conditions. The $\mathrm{AgNO}_{3}$ addition previously found to induce oxygenation like effect leading to a $T_{\mathrm{c}}$ enhancement in $\mathrm{Y}$ 123 and $\mathrm{Tl}$ doped $\mathrm{Hg}-1223$ films (Kumar et al 1993; Singh et al 1996) is not having any effect on $T_{\mathrm{c}}$. Thus in view of these observations it can be safely concluded that the role of externally supplied oxygen in the growth of Tl-
2223 is not as important as has been emphasized, especially when $\mathrm{Ba}, \mathrm{Ca}$ and $\mathrm{Cu}$ nitrates are used as the starting materials. We have also annealed the as-grown samples in flowing oxygen at $400^{\circ} \mathrm{C}$. It has been observed that the $T_{\mathrm{c}}$ decreases in both cases ( $\mathrm{Ag}$ free as well as $\mathrm{Ag}$ doped) to $\sim 110 \mathrm{~K}$ after $2 \mathrm{~h}$ of oxygenation. Even very mild oxygenation, e.g. $30 \mathrm{~min}$ at $400^{\circ} \mathrm{C}$ brings the $T_{\mathrm{c}}$ below $120 \mathrm{~K}$. This reveals that the as-grown samples in the present case have optimum oxygen content leading to near optimum $T_{\mathrm{c}}$ values.

The transport critical current density $\left(J_{\mathrm{c}}\right)$ which is an important parameter has been measured across a $0.5 \mathrm{~mm}$ bridge mechanically etched on to the film. The $J_{\mathrm{c}}$ has been defined employing the standard $1 \mu \mathrm{V} / \mathrm{cm}$ electric field criterion. The transport $J_{\mathrm{c}}$ was measured at different temperatures in the temperature range $70 \mathrm{~K} \leq T \leq 122 \mathrm{~K}$. Measurements reveal that at $77 \mathrm{~K}$ the $J_{\mathrm{c}}$ values for Ag free and $0.35 \mathrm{Ag}$ doped samples are $1.36 \times 10^{5} \mathrm{~A} / \mathrm{cm}^{2}$ and $1.72 \times 10^{5} \mathrm{~A} / \mathrm{cm}^{2}$ respectively. Clearly, there is a $\sim 25 \%$ $J_{\mathrm{c}}$ enhancement due to Ag doping. This $J_{\mathrm{c}}$ enhancement results from the fact that $\mathrm{Ag}$ goes into the grain boundaries and thus transforms the usual superconductorinsulator-superconductor (SIS) type grain configuration
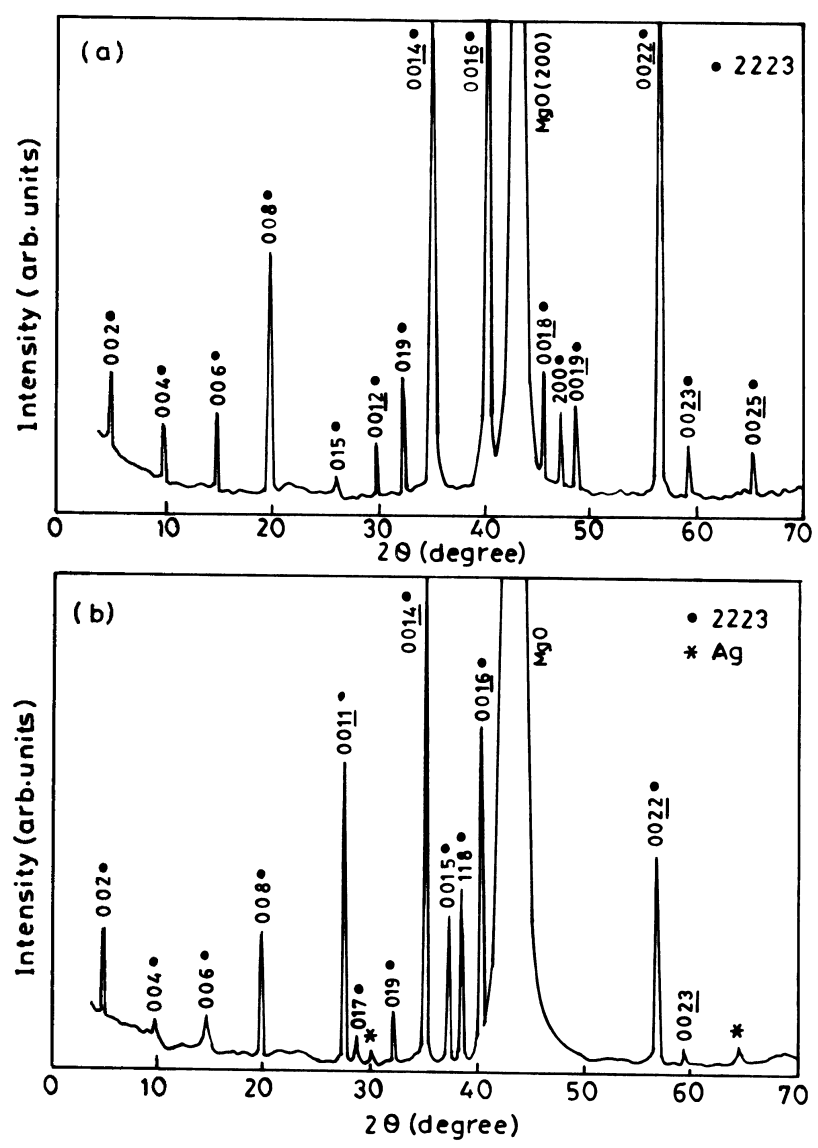

Figure 1. XRD pattern of the as-grown Tl-2223 thin films, (a) Ag free sample and (b) $0.35 \mathrm{Ag}$ doped sample. Both the $00 \mathrm{l}$ as well as other reflections have been indexed. 
into superconductor-normal metal-superconductor (SNS) type configuration. This transformation enhances intergrain current transport leading to a rise in $J_{\mathrm{c}}$. The fact that $\mathrm{Ag}$ goes into the grain-boundaries is well established (de Luca et al 1993; Kumar et al 1993; Singh et al 1995) and EDAX investigation carried out at the grain boundaries in the present case also verifies this fact.

We have also measured and evaluated the temperature dependence of $J_{\mathrm{c}}$ in the temperature range $122 \mathrm{~K}-70 \mathrm{~K}$. The results are shown in figures $4 \mathrm{a}-\mathrm{c}$ where $J_{\mathrm{c}}$ is plotted against $T$ and $1-t$, where $t=T / T_{\mathrm{c}}$. The plot in figure $4 \mathrm{a}$ shows that $J_{\mathrm{c}}$ of both $\mathrm{Ag}$ free as well as $\mathrm{Ag}$ doped films exhibits nearly similar temperature dependence. Since the temperature dependence of $J_{\mathrm{c}}$ near $T_{\mathrm{c}}$ (in the range 0.8 $T_{\mathrm{c}}<T<T_{\mathrm{c}}$ ) reflects the nature of the grain boundary junctions, we have plotted $J_{\mathrm{c}}$ against $(1-t)$ where $t=T / T_{\mathrm{c}}$. The $J_{\mathrm{c}}$ vs $(1-t)$ plot in the range $0.8 T_{\mathrm{c}}<T \leq T_{\mathrm{c}}$ $(110 \mathrm{~K}-122 \mathrm{~K})$ is shown in figure $4 \mathrm{~b}$. It has been obser-

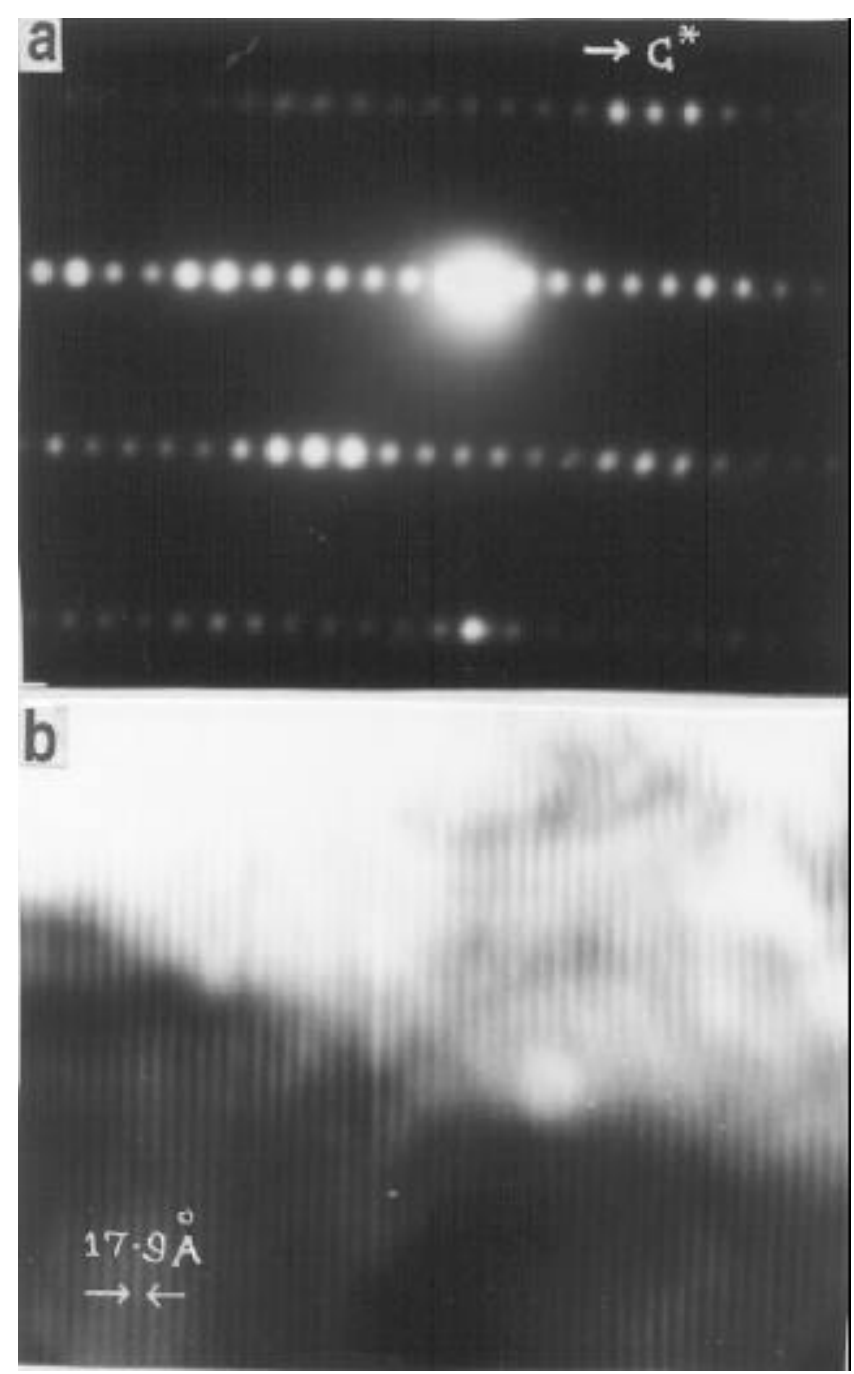

Figure 2. Representative selected area electron diffraction pattern (a) and the corresponding direct lattice image (b). ved that in this region $J_{\mathrm{c}}$ of both $\mathrm{Ag}$ free as well as $\mathrm{Ag}$ doped films follows a power law of the type $J_{\mathrm{c}} \propto(1-t)^{p}$. The value of the exponent for Ag free sample is $p=1.92$ and for Ag doped sample $p=1.96$. This near equality of exponents and the fact that $p \approx 2$ suggests that in both cases samples have SNS type grain boundary configuration (de Gennes 1964; Kumar et al 1995). However, this may not be the case and the observed data can alternatively be explained as follows. It has been suggested (Deutscher and Müller 1987) that in HTSCs a power law variation of the type $J_{\mathrm{c}} \propto(1-t)^{2}$ can occur at temperatures close to $T_{\mathrm{c}}$ in both SIS and SNS type configurations. This is because in HTSC cuprates the zero temperature coherence length $\xi(0)$ is very small as compared to the conventional superconductors. Consequently in these cuprates the insulating regions will result in SIS type junctions with a depressed order parameter at the junction interfaces. In such cases, as pointed out by Deutscher and Muller (1987) a power law of the type $J_{\mathrm{c}} \propto(1-t)^{2}$ follows. Thus it can be said that in the present case $\mathrm{Ag}$ free samples have SIS type grain boundary configuration even when $J_{\mathrm{c}} \propto(1-t)^{1.92}$. In the case of Ag doped samples, Ag migrates into the grain boundaries leading to a transformation of SIS type junctions to SNS type. In this case the normal metallic region between the superconducting grains will result in proximity effect SNS junctions, giving a $J_{\mathrm{c}}$ dependence, again of the type $J_{\mathrm{c}} \propto(1-t)^{2}$. The observed value of the exponent ( $p=1.96)$ for $\mathrm{Ag}$ doped films is close to 2. Thus our experimental findings are well in agreement with the

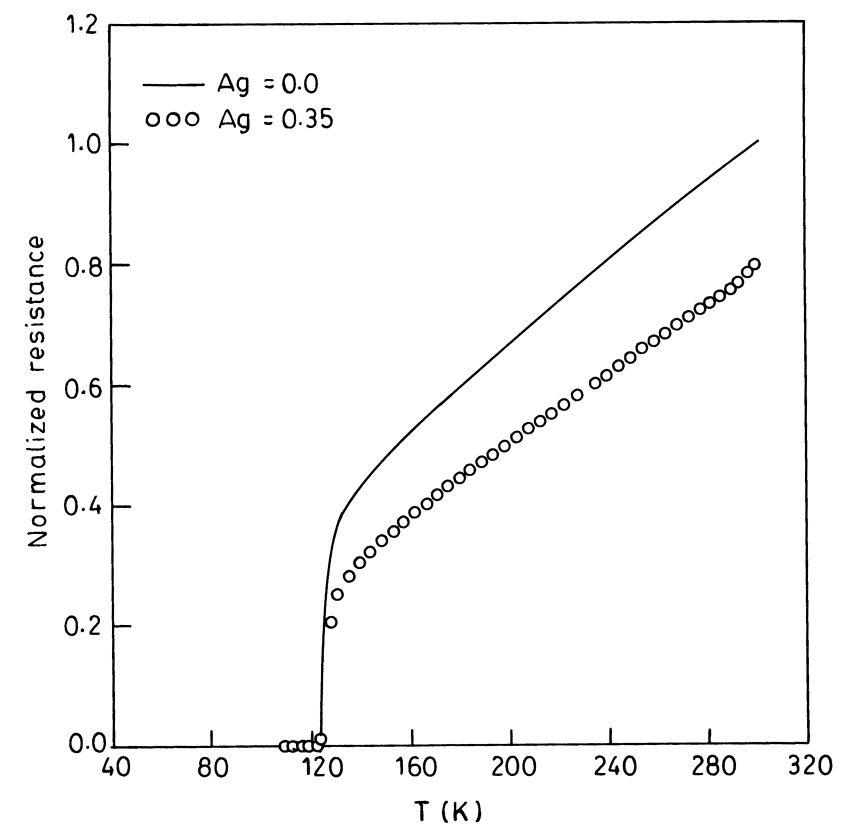

Figure 3. $R-T$ characteristics of the as-grown $T 1-2223$ thin films. The observed temperature dependence of resistance in the normal state as well as the nature of transition in both the samples is remarkably similar. 

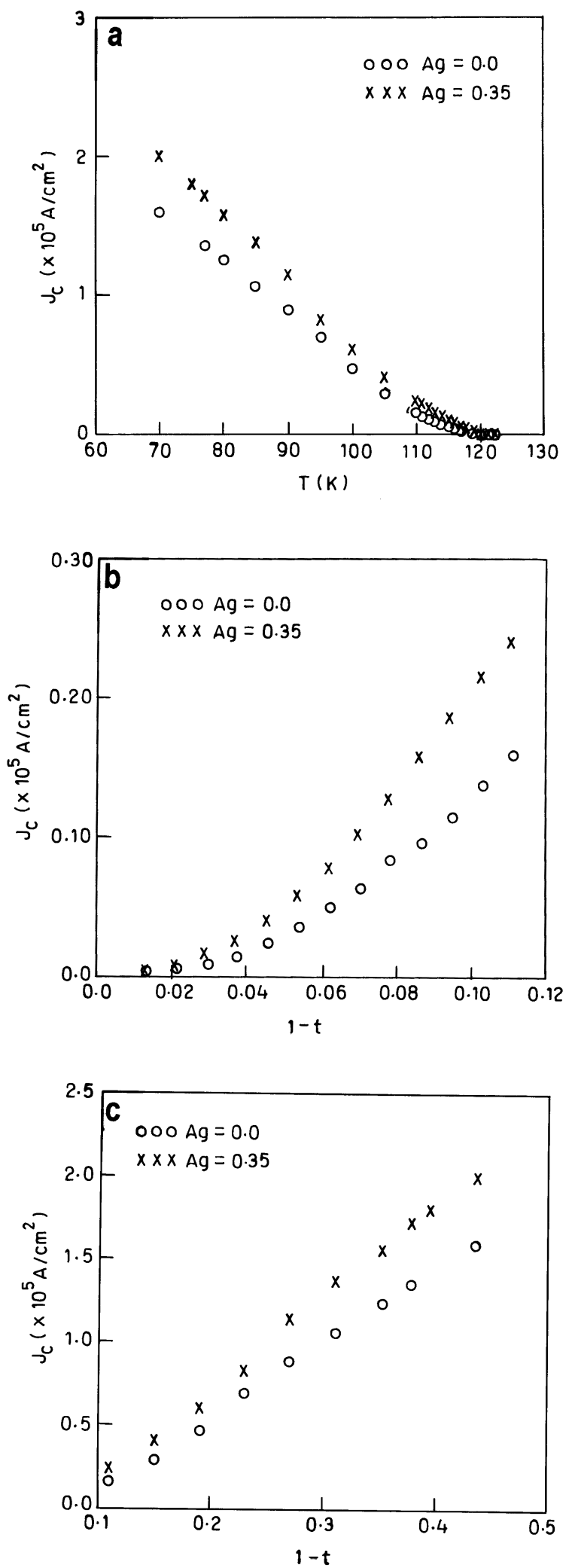

Figure 4. Temperature dependence of critical current density $\left(J_{\mathrm{c}}\right)$ of silver free and silver doped samples: a. variation of $J_{c}$ with $T$ for as-grown $\mathrm{Ag}$ free and $0.35 \mathrm{Ag}$ doped sample in the temperature range $122 \mathrm{~K}-70 \mathrm{~K}, \mathbf{b}$. variation of $J_{\mathrm{c}}$ with $1-t$, $t=T / T_{\mathrm{c}}$ in the temperature range $122 \mathrm{~K}-110 \mathrm{~K}$ and c. variation of $J_{\mathrm{c}}$ with $1-t, t=T / T_{\mathrm{c}}$ in the temperature range $110 \mathrm{~K}-70 \mathrm{~K}$. theoretical model of Deutscher and Müller (1987). In the lower temperature range, i.e. from $110 \mathrm{~K}$ to $70 \mathrm{~K}, J_{\mathrm{c}}$ still follows a power law. But in this temperature regime the value of the exponent $p$ is reduced and in fact $p$ lies close to 1.4 . The observed variation is shown in figure $4 \mathrm{c}$.

The SEM aided surface topographic evaluation of the $\mathrm{Ag}$ free and $0.35 \mathrm{Ag}$ added samples reveal some interesting features. The representative SEM micrographs are shown in figure 5. In case of $\mathrm{Ag}$ free as well as $0.35 \mathrm{Ag}$ doped samples plate like grains are seen (figures $5 a, b$ ). But in the Ag free sample grains have varying sizes and their distribution is non-uniform (figure 5a). On the other hand in $0.35 \mathrm{Ag}$ doped samples, the growth of uniform size grains is seen (figure $5 b$ ). The grains are nearly rectangular and a grain approximately measures $15 \times 10 \mu \mathrm{m}^{2}$.

From the growth point of view the SEM investigation shows that Ag doping leads to enlarged and uniform grain growth. This is possibly brought about by improved nucleation and growth kinetics induced by highly mobile Ag particles. Silver particles act as nucleation centres and once the nucleation is complete and growth starts, Ag atoms are unable to sit at any of the Tl-2223 lattice sites and come out of the growing superconducting grain. When these highly energetic and mobile $\mathrm{Ag}$ particles come out of the growing superconducting grain they possibly also define the grain size and shape. This shape in the present case is nearly rectangular (figure $5 \mathrm{~b}$ ). Since the growth temperature is higher than the melting temperature of $\mathrm{Ag}$, the presence of molten silver in $0.35 \mathrm{Ag}$ doped samples greatly reduces the surface tension between the grain and the substrate during growth. This leads to better grain orientation and well defined grain shape. These observations are well in agreement with the model proposed by Kumar et al (1995). SEM investigation also shows that the individual grains themselves consist of very fine layers. This is true for both, $\mathrm{Ag}$ free as well as Ag doped samples. Another important microstructural feature observed in the SEM investigations is that in some plate like grains these fine structure layers take the form of a polygonal spiral. These spirals give the impression that they are formed over the plate like grains. Single loop and closed loop spirals have been observed. However, it must be mentioned that the growth of these features is only sporadic and such features have been observed in both, Ag free as well as 0.35 Ag doped samples. A typical polygonal shaped spiral is shown in figure $5 \mathrm{c}$. Thus the microstructure of the Tl-2223 films consists of (i) plates having very fine layers but no spirals and (ii) plates having polygonal shaped spirals. The grains having spirals may have their origin in the screw dislocations in the substrates or instantaneously created screw dislocations during the growth. The occurrence of spiral features is only sporadic making it very difficult to have any clear idea regarding their density. 

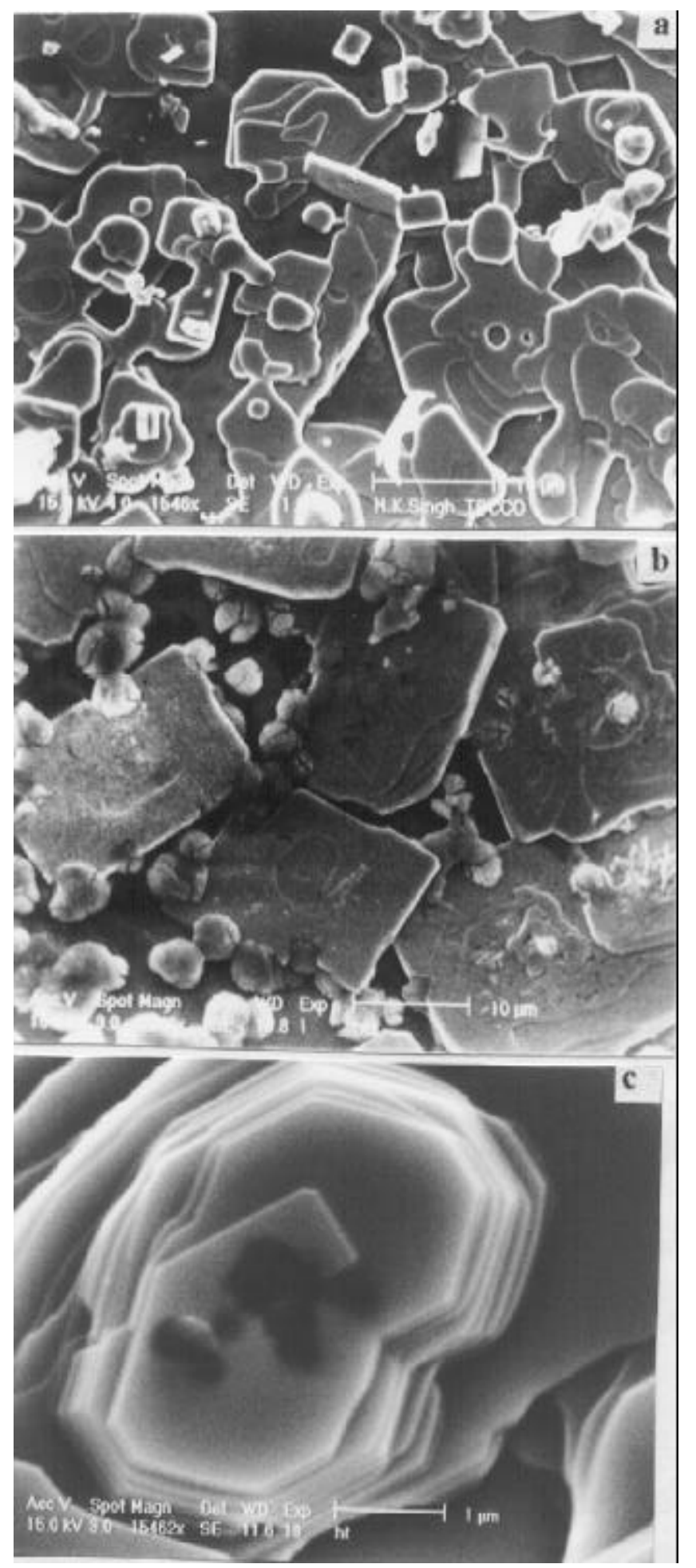

Figure 5. Representative SEM micrographs showing the surface morphology of a. as grown Ag free sample and b. $0 \cdot 35$ Ag doped sample. c. depicts SEM micrograph taken from $0 \cdot 35 \mathrm{Ag}$ added sample showing polygonal shaped spiral growth feature.

\section{Conclusions}

In the present investigation single phase, $\mathrm{Ag}$ free and 0.35 $\mathrm{Ag}$ doped Tl-2223 thin films have been grown on single crystal $\mathrm{MgO}$ substrates. In the present investigation the thalliation of the precursor films has been carried out in high vacuum. This is in contrast to previous studies which have used oxygen. The oxygen deprived conditions have been found to be conducive to the growth of the Tl-2223 phase possessing high $T_{\mathrm{c}}$ and $J_{\mathrm{c}}$. In fact the $T_{\mathrm{c}}=123.7 \mathrm{~K}$ observed in the present case is the highest observed $T_{\mathrm{c}}$ so far for the virgin Tl-2223 films. The $J_{\mathrm{c}}$ values $>1 \times 10^{5} \mathrm{~A} / \mathrm{cm}^{2}$ $(77 \mathrm{~K})$ are also quite encouraging for the spray pyrolysis technique. In the present work it has been shown that oxygen ambient is not necessary for the growth of Tl2223 films, especially when the nitrates are taken as the starting materials. Effect of $\mathrm{Ag}$ has been positive on $J_{\mathrm{c}}$ and microstructure and $T_{\mathrm{c}}$ has remained broadly unaffected. The Ag doping has been found to profoundly change the grain size and evolve a uniform grain size distribution. The plate like superconducting grains have been observed to be composed of very thin layers themselves. Thus it is obvious from the above detailed and discussed results that the present route is advantageous in several ways. This route is quite safe as thalliation is carried out in closed and sealed chambers. Materials loss, especially of $\mathrm{Tl}_{2} \mathrm{O}_{3}$ is minimized due to closed chamber thalliation. The present route yields films of good quality as evidenced by structural/microstructural and electrical characteristics described above.

\section{Acknowledgements}

Authors are grateful to Professors A R Verma, C N R Rao, FRS, T V Ramakrishnan, FRS, G V Subba Rao, A V Narlikar and Dr K Lal for several inspiring discussions. Authors are also grateful to Dr A M Herman, University of Colorado, Boulder, USA for valuable discussion during his visit to our laboratory. One of the authors (HKS) thankfully acknowledges the University Grants Commission, New Delhi for financial assistance in the form of Junior and Senior Research Fellowships.

\section{References}

de Luca J A, Karas P L, Tkaczyk J E, Bednarczyk P I, Garbauskas M F, Braint C L and Sorensen B D 1993 Physica C205 21

de Gennes P G 1964 Rev. Mod. Phys. 36225

Deutscher G and Müller K A 1987 Phys. Rev. Letts 591745

Holstein W L and Parisi L A 1996 J. Mater. Res. 111349

Jergel M 1995 Supercond. Sci. Technol. 867

Khare N, Gupta A K, Saxena A K, Verma K K and Srivastava O N 1994 Supercond. Sci. Technol. 7402

Khare N, Gupta A K, Khare S, Singh H K, Saxena A K and Srivastava O N 1997 Physica C274 161 
Kumar D, Sharon M, Pinto R, Apte P R, Pai S P, Purandare S P, Gupta L C and Vijayaraghavan R 1993 Appl. Phys. Letts 623552

Kumar D, Apte P R and Pinto R 1995 J. Appl. Phys. 775802

Lee W Y 1990 Appl. Phys. Letts 60772

Lee W Y, Lee V Y, Salem J, Huang T C, Savoy R, Bullock D C and Parkin S S P 1989 Appl. Phys. Letts 59329

Liu R S, Tallon J L and Edwards P P 1992 Physica C182 119

Mayers K E, Face D W, Kuntz K J and Nestlerode J P 1994 Appl. Phys. Letts $\mathbf{6 5} 490$

Moriwaki Y, Sugano T, Grasser C, Fukuoka A, Nakanishi K, Adachi S and Tanabe K 1996 Appl. Phys. Letts 693423
Nabatame T, Saito Y, Aihara K, Kamo T and Matsuda S P 1990 Jap. J. Appl. Phys. 29 L1813

Narain S and Rukenstein A E 1989 Supercond. Sci. Technol. 2236

Siegal M P, Venturjmi E L, Morision B and Aselage T L 1997 J. Mater. Res. 122885

Singh H K, Saxena A K and Srivastava O N 1995 Supercond. Sci. Technol. 8448

Singh H K, Saxena A K and Srivastava O N 1996 Physica C262 7

Singh H K, Saxena A K and Srivastava O N 1997 Physica C273 181

Su L Y, Grovener C R M, Goringe M S, Deuhurst C D, Cardwell D A, Jenkins R and Jones H 1994 Physica C229 70 